\title{
Shaping the Future
}

To create shape-shifting robotic ensembles, researchers

need to teach micro-machines to work together.

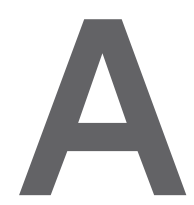

PROTOTYPE OF yOur company's latest product sits on the conference table. You suggest sleeker styling. The 3D model reacts, fluidly changing its angles. A colleague recommends different proportions, and again the model reflects those edicts. A pass of the hands changes the model's color; another reveals its interior, while a third folds it back into a flat sheet. Computer-aided design software records all the changes to replay them later, and to easily produce the final version.

That's the vision of researchers at Carnegie Mellon University and elsewhere as they embark in the new field of robotics known as claytronics. In it, a shape-shifting object is comprised of thousands of miniature robots known as claytronic atoms, or catoms. Each catom has the power to move, receive power, compute, and communicate with others, resulting in an ensemble that dynamically envisions three-dimensional space much as the pixels on a computer monitor depict a two-dimensional plane. Functional scenarios for claytronics include a general-purpose computer changing from laptop to cellphone form as needed, or, say, a plumber's tool that changes from a scuttling, insect-like device in crawl spaces to a slithering snake-like shape when inside pipes.

Ultimately, the claytronics dream goes, we may have morphing humanoids like T-1000 in the movie Terminator 2 or Odo in the TV series "StarTrek: Deep Space Nine." Because their shape-shifting abilities could make them excellent mimics of human forms, such androids might act as reusable stand-ins for surgeons, repair technicians, and other experts who control them remotely.

\section{Collaborative Research}

Two joint ventures have taken the lead in this field. One partnership-

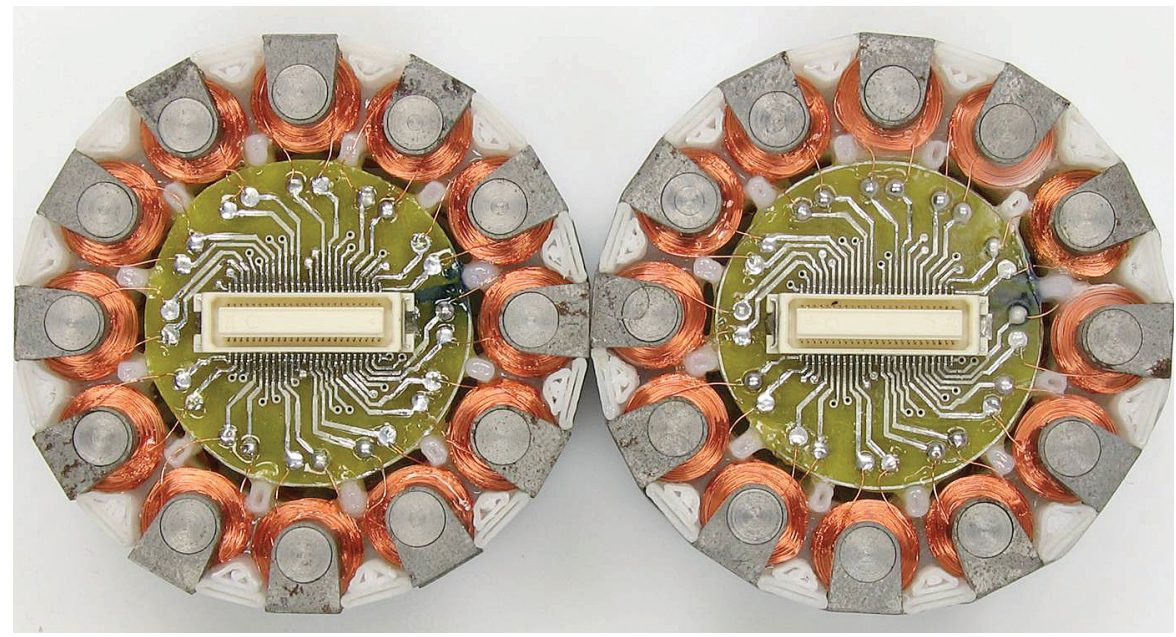

A top view of two magnet rings, with individual driver boards, from the self-actuating Planar Catom V7 developed by the Carnegie Mellon University-Intel claytronics research team.

between Carnegie Mellon University's Collaborative Research in Programmable Matter project and Intel Research Pittsburgh's Dynamic Physical Rendering project-focuses on programmable materials' shape-shifting aspects as well as the software needed to drive it. The other major effort eyes military applications; this collaboration is between the Defense Advanced Research Projects Agency (DARPA) and a consortium of colleges including the University of California at Berkeley, Harvard University, the University of Pennsylvania, Massachusetts Institute of Technology, and Cornell University.

\section{To form stable ensembles, catoms must have power, communication, and adherence to one another.}

Both projects face a host of challenges. Most immediately, the individual mechanical devices need to be much smaller than the units most recently demonstrated, which were about the size of large salt shakers. Jason Campbell, a senior staff research scientist at Intel's Pittsburgh laboratory, says his group is working with catoms of a tubular structure that are much smaller-1 millimeter in diameter and 10 millimeters in length. He believes that they need only be small enough to disappear to our eyes and touch. "That gives us a size target between a tenth of a millimeter and one millimeter across," he says, with the size requirement depending on application. A shape-changing radio antenna doesn't need to be very small, for example, whereas for a handheld device, "the consumer is the perceptual system" and miniaturization has great value, says Campbell.

Claytronics research flows out of the field of modular robotics, where individual units are typically a few inches across. But as the units get smallerand more numerous - the physics controlling them changes. Kasper Støy, associate professor of computer systems engineering at the University of South- 
ern Denmark, points to one difference between the macro and micro levels. With larger modules, he says, "we have to fight a lot with gravity. All the modules have to be able to lift the others. We've long used electromechanical actuators to overcome gravity in large modules. But at the micro level, you don't want to have a big actuator in every little unit. The question is, How do you go from a bunch of really weak modules and get a big, strong robot?"

Size is not the only challenge. To form stable ensembles, catoms must have power, communication, and adherence to one another. The Carnegie Mellon project is currently attempting to address most of these issues by attaching electrostatic plates to the surface of each catom. Plates given opposite charges would cling to each other; changing the charges in a precisely programmed sequence would force the plates to roll over one another into the desired configuration. Information could be exchanged between adjoining catoms by manipulating their voltage differences.

Claytronics researchers are considering several options for providing power. In early versions, "catoms will get power from the table they sit on, through capacitive coupling," says Seth Goldstein, an associate professor of computer science who leads the Carnegie Mellon team. But additional technologies are also under consideration, Goldstein says. "We are looking at magnetic resonance coupling, which goes over a longer distance," he says. "We're also looking at solar power. Because of their size, the catoms are translucent. My guess is that we'll use multiple ways of getting power in."

Mark Yim, an associate professor of mechanical engineering at the University of Pennsylvania who is involved with the DARPA project, has experimented with drawing power from the environment and letting the catoms' intelligence and adhesion turn it into useful motion. "I asked, What if we shake the table to get the modules to move around each other, and the modules determine when to grab or let go?" says Yim. "If they get really small, you might be able to move them with sound waves that shake the air."

As catom production ramps up, these questions assume greater urgency. Goldstein notes that his lab, in collaboration with the U.S. Air Force Research Laboratory, has "refined the process to print the catom's circuits on a standard silicon die, then postprocess it to curl up into a sphere or a tube." By this December, Goldstein says, he hopes to fabricate a silicon cylinder about one millimeter in diameter that will "move around under its own control, transfer power from a ground plane, and be able to communicate with other units."

\section{The Shapes to Come}

A pile of catoms is useless without coordination. And although the challenge of creating claytronic software is similar to that of any massively parallel computer system, there is one key difference: The catoms' physical nature makes errorcorrection more important. "Catoms

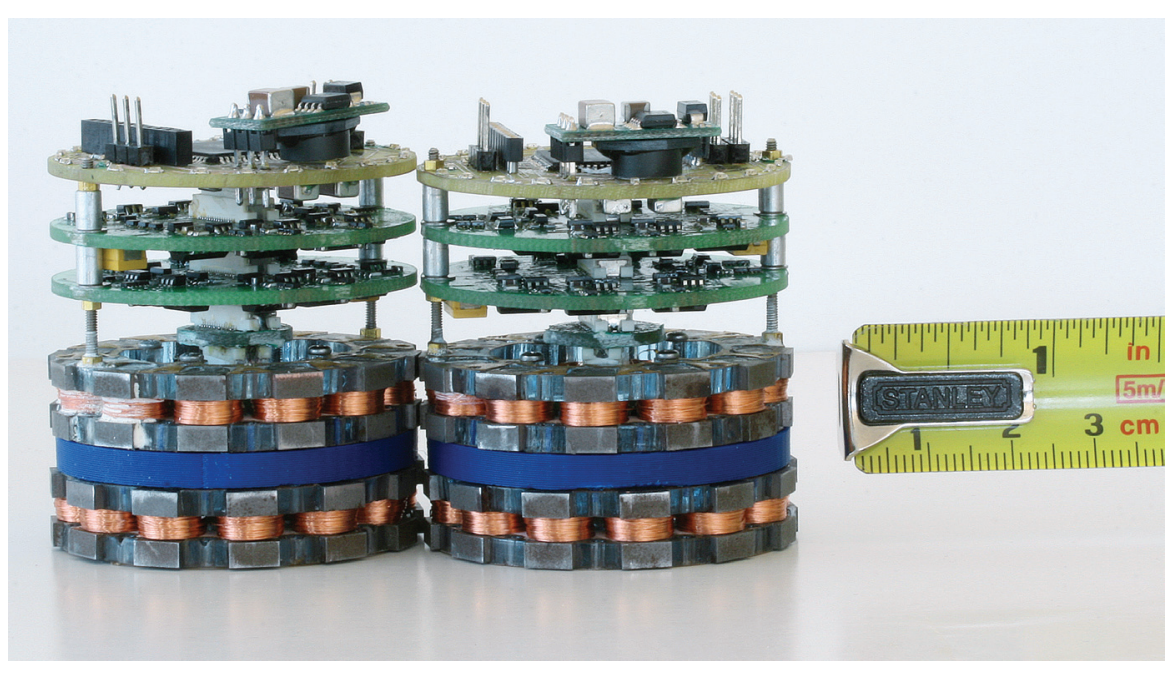

A pair of Planar Catom V8s, each of which weighs 100 grams, with their stack of magnetsensor rings on the bottom and solid-state electronic control rings on the top.
Education

\section{U.S. CS Courses Decline}

The number of computer science courses being taught in high schools throughout America is steadily decreasing, according to the 2009 CSTA Secondary Computer Science Survey conducted by the Computer Science Teachers Association. The survey, based on responses from 1,094 high school teachers in spring 2009 , found that only $65 \%$ of schools offer an introductory CS course, compared with $73 \%$ in 2007 and $78 \%$ in 2005 . Likewise, the number of advanced placement CS courses has also declined, with $27 \%$ in $2009,32 \%$ in 2007 , and $40 \%$ in 2005 .

"There are really three big requirements for reversing the trends highlighted in the survey," said CSTA Director Chris Stephenson in an email interview. "First, we need to ensure that computer science is classified as a rigorous academic subject in $\mathrm{K}-12$ and not as a 'technical' or 'vocational' subject. Second, as a community, we have to do a much better job of explaining to policymakers, parents, and students what unique skills and knowledge computer science provides and what future opportunities it offers. Finally, we have to fix the mess that is computer science teacher preparation and certification in most states, so that computer science teachers can be certified as CS teachers and not as math, science, business, or tech teachers.

"Our highly decentralized education system in the U.S. makes it very difficult to achieve systematic, long-term change," said Stephenson. "In each state, the players, the priorities, and the policies are different."

The survey's findings were not unexpected. "This is the third time we have done this survey," said Stephenson, "and so while we were not really surprised by the results, we were more troubled than ever with the continued decline in the number of courses being offered and the fact that it is getting increasingly harder for students to fit elective computer science courses into their timetables." 


\section{For catoms to be commercially viable, large quantities of the devices must be produced at a low cost.}

are mechanical devices, and mechanical devices fail," says Campbell.

There's also the question of external control: How much instruction should the aggregate get from an outside source? Boston University electrical and computer engineering professor Tommaso Toffoli expresses skepticism about the ability of a claytronic ensemble to be self-directed. In the 1990s, he says, companies attempting to build massively parallel computers failed because they misread this problem. "People eventually found that instead of having millions of tiny computers the size of ants trying to simulate the brain, they got more work done by having just a few hundred that act as slaves, carrying sand and water to a floating-point processor," Toffoli says.

Campbell disagrees. He believes that for catoms to be as effective as possible, they will each need to have onboard intelligence and that process- ing tasks will need to be distributed throughout the ensemble. "The more parts you have, the more it matters that you have processing on each part. There are still things you can centralize, but decisions about what modules to move when have to be done inside the aggregate," says Campbell.

The Carnegie Mellon team uses simulations to address these questions, and has created two languages that are optimized to program the ensemble. One, called Meld, is a stateless, declarative, logic-based language used to control the behavior of individual catoms. The second, locally distributed predicates (LDP), is used to recognize patterns and evaluate conditions throughout the ensemble as a whole. Meld and LDP are complementary, says language developer Michael Ashley-Rollman, a computer science graduate student at Carnegie Mellon. "If one robot stops working," he explains, "Meld just throws everything away from that robot and pretends that it was never there." LDP, on the other hand, "permits programmers to manage states themselves"-at the expense of error correction. LDP, Ashley-Rollman says, "gives Meld rules through which it can determine what the state can be."

\section{The Reality Challenge}

Commercial viability for catoms will require major advances in the ability to produce large quantities of the devices at low cost. Goldstein hopes that his lab at Carnegie Mellon will build working, submillimeter catoms by the end of 2009. By 2012, he believes, the researchers will have "hundreds of catoms working together intelligently." More fantastically, Goldstein hopes that in 30 years there will be a claytronic humanoid that looks, feels, and moves in ways almost identical to a person.

But such ambitious goals may obscure a more fundamental issue. "Say, you have something like claytronics," Yim says. "You have the mechanical part working. You've got the computational part working so you can control them in a distributed fashion. The question is, What do you do with it? Maybe you want it to go get a beer from the refrigerator. How does it know what is the best shape to be in to open the door?"

Indeed, making claytronics truly useful depends on developments in areas beyond these projects' current scope, including artificial intelligence and human modeling. But the scenarios described only illustrate possible applications for claytronics, and in fact researchers are quicker to say what the technology will be than what it will do. But that's the very problem that general-purpose computers faced until the mid-1980s; like PCs, claytronic ensembles may ultimately prove their worth in ways now unimagined.

Tom Geller is an Oberlin, Ohio-based science, technology, and business writer.

(C) 2009 ACM 0001-0782/09/1000 \$10.00

\section{Minestones}

\section{Computer Science Awards}

The Internet Society and the National Science Foundation (NSF) recently honored members of the computer science community for their innovative research.

\section{POSTEL SERVICE AWARD} The Internet Society awarded the 2009 Jonathan B. Postel Service Award to CSNET, the research networking effort that during the early 1980 s provided the critical bridge from the original research undertaken through ARPANET to today's Internet. Four principal investigators-Peter J. Denning,
David Farber, Anthony C. Hearn, and Lawrence Landweber-and NSF program officer Kent Curtis, who encouraged and funded CSNET, were recognized for their important roles.

CSNET began in 1981 with a five-year NSF grant, and by 1986 CSNET had connected more than 165 academic, government and industry computer research groups, which comprised more than 50,000 researchers, educators and students around the world. Open to computer researchers, CSNET, which was self-governing and self- supporting, demonstrated that researchers valued the type of informal collaboration it made possible. The success of CSNET encouraged NSF to undertake the NSFNET program, which brought open networking to a larger academic community and presaged the emergence of today's Internet.

\section{NSF CAREER AWARDS}

NSF recently awarded Mathews Jacob, an assistant professor in the department of biomedical engineering at the University of Rochester, and Haijun Su, an assistant professor in the University of Maryland, Baltimore County's mechanical engineering department, with Faculty Early Career Development Awards. Jacob was recognized for his research into the design of computer programs that accelerate the capture of highresolution images, and that promise to enable early diagnosis of cancer. Su's virtual reality research involves the creation of a virtual design environment in which conceptual designs can be quickly and inexpensively evaluated. 